\title{
Diagnostic value of 64-slice spiral computed tomography imaging of the urinary tract during the excretory phase for urinary tract obstruction
}

\author{
DE-LI ZHAO ${ }^{1 *}$, GUANG-SHENG JIA $^{1 *}$, PENG CHEN $^{2}$, XIN-DING LIU ${ }^{1}$, SHENG-JIE SHU $^{1}$, \\ ZAI-SHENG LING ${ }^{1}$, TING-TING FAN ${ }^{1}$, XIU-FEN SHEN ${ }^{1}$ and JIN-LING ZHANG ${ }^{1}$ \\ ${ }^{1}$ Department of Computed Tomography, The Second Affiliated Hospital of Harbin Medical University, Harbin, \\ Heilongjiang 150086; ${ }^{2}$ Radiology Department, The Fourth Hospital of Harbin, Harbin, Heilongjiang 150026, P.R. China
}

Received November 1, 2016; Accepted June 29, 2017

DOI: $10.3892 /$ etm.2017.5176

\begin{abstract}
The present study aimed to assess the diagnostic value of 64-slice spiral computed tomography (CT) imaging of the urinary tract during the excretory phase for urinary tract obstruction. CT imaging of the urinary tract during the excretory phase was performed in 46 patients that had been diagnosed with urinary tract obstruction by B-mode ultrasound imaging or clinical manifestations. It was demonstrated that out of the 46 patients, 18 had pelvic and ureteral calculi, 12 cases had congenital malformations, 3 had ureteral stricture caused by urinary tract infection and 13 cases had malignant tumors of the urinary tract. The average X-ray dose planned for the standard CT scan of the urinary tract group 1 was $14.11 \pm 5.45 \mathrm{mSv}$, while the actual X-ray dose administered for the CT scan during the excretory phase group 2 was $9.01 \pm 4.56 \mathrm{mSv}$. The difference between the two groups was statistically significant $(\mathrm{t}=15.36 ; \mathrm{P}<0.01)$. The results of the present study indicate that $\mathrm{CT}$ scanning of the urinary tract during the excretory phase has a high diagnostic value for urinary tract obstruction.
\end{abstract}

\section{Introduction}

The use of 64-slice spiral computed tomography (CT) imaging of the urinary tract provides the benefits of fastness, a wide scan range, high $\mathrm{Z}$-axis resolution and little interference from the surrounding organs, such as the intestinal tract (1).

Correspondence to: Dr Jin-Ling Zhang, Department of Computed Tomography, The Second Affiliated Hospital of Harbin Medical University, 245 Xuefu Street, Harbin, Heilongjiang 150086, P.R. China

E-mail: zjinlzjl@126.com

*Contributed equally

Key words: X-ray computed tomography, urography, urinary tract disease
This imaging technique is unparalleled for the diagnosis and three-dimensional (3D) display of urinary tract obstruction $(2,3)$. With a standard CT scan of the urinary tract, images are captured during the arterial, parenchymal and excretory phases, and a full-range non-enhanced scan may be required at times. This not only prolongs the total scan time and therefore time spent in the CT scanner, but also exposes the patient to a higher X-ray dose (4). However, single scan radiation dose is small and imaging time is short, and is therefore a feasible operation (5). Based on B-mode ultrasound or clinical manifestations, 46 cases of urinary tract obstruction with unknown causes were selected for inclusion in the current study. Patients received CT scans with different delay times during the excretory phase. The aim of the current study was to assess the single excretory phase diagnostic value of 64-slice spiral CT for urinary tract obstruction.

\section{Materials and methods}

Clinical data. Between January 2013 and January 2015, urinary tract obstruction of varying degrees was diagnosed by B-mode ultrasound imaging and clinical manifestations, including back pain and hematuria, in 46 cases at Department of CT, the Second Affiliated Hospital of Harbin Medical University (Harbin, China). Of the 46 patients, 32 were male and 21 were female. The patient's age ranged from 6 to 71 years old. A total of 43 patients reported lower back pain and 32 patients exhibited hematuria. The study included patients who had urinary obstruction, and excluded patients who had undergone kidney transplantation, dialysis or were allergic to contrast agents. The present study was approved by the Institutional Review Board of the Second Affiliated Hospital of Harbin Medical University (Harbin, China) and written informed consent was obtained from each patient after the nature of the procedure had been fully explained.

Examination method and scan preparation. The patients drank $200 \mathrm{ml}$ of water prior to the CT scans. Then, using a high-pressure injector, 70-90 ml of non-ionic contrast agent was injected at a rate of 2-3 ml/sec via the medial cubital vein. Depending on the severity of the urinary tract obstruction, 
as indicated by B-mode ultrasound, the urinary tract was subjected to a single 64-slice spiral CT urography during the excretory phase for 20-30, 30-60 or 50-180 min for mild, moderate and severe conditions, respectively. In combination with renography, the scan delay time was increased for patients with poor renal function. According to the extent of the renal index, in which the damage ranged from 20 to $45 \%$, the scan delay time was increased by $30-60 \mathrm{~min}$ as described previously (2-4).

The severity of hydronephrosis was classified based on the B-mode ultrasound results according to the following criteria: Mild hydronephrosis, a dilation of one or more renal calyces with mild pyelic separation and pyelectasia, but without parenchymal thinning under compression; moderate hydronephrosis, a dilation of all renal calyces with parenchymal thinning under compression, but still maintaining a thickness $>5 \mathrm{~mm}$; severe hydronephrosis, a dilation of all renal calyces with parenchymal thinning and a thickness $<5 \mathrm{~mm}$.

Scanning method. To perform scans on group $1(n=46)$, a GE Light Speed VCT 64-slice CT scanner (GE Healthcare, Chicago, IL, USA) was used (120 kV, 200-380 mA). Three scan sequences of the standard scan were obtained for the arterial, parenchymal and excretory phases. For the first two phases, the scan range only consisted of the kidneys; for the excretory phase, the scan was performed from the kidneys to the symphysis pubis. The two sequences obtained during the arterial and parenchymal phases were deleted after the X-ray dose administered was measured. Only the excretory phase scan was used for group $2(n=46)$.

Image reconstruction. All original images were uploaded to the workstation for imaging analysis. The reconstruction method included volume-rendering (VR), maximum intensity projection (MIP), multi-planar reformating (MPR), and curved planar reformating (CPR) using standard procedures (2).

Statistical analysis. Continuous variables were expressed as the mean \pm standard deviation and categorical variables were expressed as frequency or percentage. $\mathrm{P}<0.05$ was considered to indicate a statistically significant difference. All statistical analysis was performed by using commercially available software (SPSS18.0; SPSS, Inc., Chicago, IL, USA).

\section{Results}

Patient diagnosis. The excretory phase scan indicated all the patients suffered from ureter obstruction, and the diagnosis rate was $100 \%$ compared with clinical outcomes including surgery and ureteroscopy. Of the 46 patients in the present study, the obstructed ureter was fully displayed in the first single excretory phase CT urography with good continuity in $96 \%$ of cases. The remaining $4 \%$ patients were successfully confirmed with ureter obstruction after the second excretory phase CT scan. Of the 46 patients with pelvic and ureteral diseases, 18 had pelvic and ureteral calculi (Fig. 1). In addition, 12 patients had congenital malformations; double pelvis and ureters in 5 cases (Fig. 2), horseshoe kidneys in 2 cases (Fig. 3), ureteral valve disease in 2 cases (Fig. 4), congenital mega ureter in 2 cases (Fig. 5) and ureteropelvic junction tortuosity and stricture in
1 case. A total of 3 patients exhibited a ureteral stricture caused by urinary tract infections (urinary tuberculosis in 2 cases and inflammatory stricture in 1 case). Furthermore, 13 patients had malignant tumors of the urinary tract, including pelvis or ureter cancer in 9 cases (Figs. 6 and 7) and bladder cancer with ureteral involvement in 4 cases (Fig. 8).

Radiation dosages. The average X-ray dose planned for the standard CT scan of the urinary tract was $14.11 \pm 5.45 \mathrm{mSv}$, while the actual X-ray dose administered for CT scan during excretory phase was $9.01 \pm 4.56 \mathrm{mSv}$ (data not shown). This difference was of statistical significance between groups 1 and $2(\mathrm{t}=15.36 ; \mathrm{P}<0.01)$.

\section{Discussion}

Multi-slice spiral CT scanning of the urinary tract has been identified to have wide applications in the clinics (6). Conventional procedures require an extensive scan scope, a considerable number of scan sequences and a long duration of patient presence in the CT scanner. Furthermore, patients are exposed to a high X-ray dose using standard CT imaging. Reducing the duration of scanning and the X-ray dose that the patient is exposed to during CT imaging of the urinary tract are research topics that are increasingly drawing attention (7-9).

CT imaging of the urinary tract can be applied to diagnose lesions in any part of the urinary system, including the kidneys, ureter and bladder. Of the various benefits displayed by multi-slice CT technique for the diagnosis of urinary tract obstruction, 3D display of the excretory phase is of particular benefit (6). However, single scan radiation doses are small and the imaging time is short; therefore, this technique is feasible (6). By allocating a different scanning time (30-60 min) for different severities of urinary tract obstruction, the delay time is prolonged for those with poor renal function based on renography results. Of the 46 patients with pelvic and ureteral diseases, CT scanning during the excretory phase diagnosed 18 cases of pelvic and ureteral calculi, 12 cases of congenital malformations (double pelvis and ureters in 5 cases, horseshoe kidneys in 2 cases, Ureteral valve diseasein 2 cases, congenital megaureter in 2 cases and ureteropelvic junction tortuosity and stricture in 1 case). In addition, 3 patients exhibited ureteral stricture caused by urinary tract infections (urinary tuberculosis in 2 cases and inflammatory stricture in 1 case), 13 patients had malignant tumors of the urinary tract (pelvis or ureter cancer in 9 cases and bladder cancer with ureteral involvement in 4 cases). The diagnosis rate of urinary tract obstruction was $100 \%$ for all patients at the obstruction sites, diseased sites and the full length of ureter on the affected side. The average X-ray dose planned for the standard CT scan of the urinary tract was $14.11 \pm 5.45 \mathrm{mSv}$, and the actual $\mathrm{X}$-ray dose administered for CT scan during excretory phase was $9.01 \pm 4.56 \mathrm{mSv}$. CT scanning was performed at a standard voltage of $120 \mathrm{kV}$ for all patients. Compared with conventional imaging procedures, $\mathrm{CT}$ scanning during the excretory phase alone reduces the scanning time and $\mathrm{X}$-ray dose by $>30 \%$ (6). However, this reduction would be increased at a lower $\mathrm{kV}$ value, as indicated by a previous report (2).

B-mode ultrasound and intravenous pyelogram (IVP) imaging are common tools for diagnosing urinary tract obstruction, with the benefits of being simple procedures that 


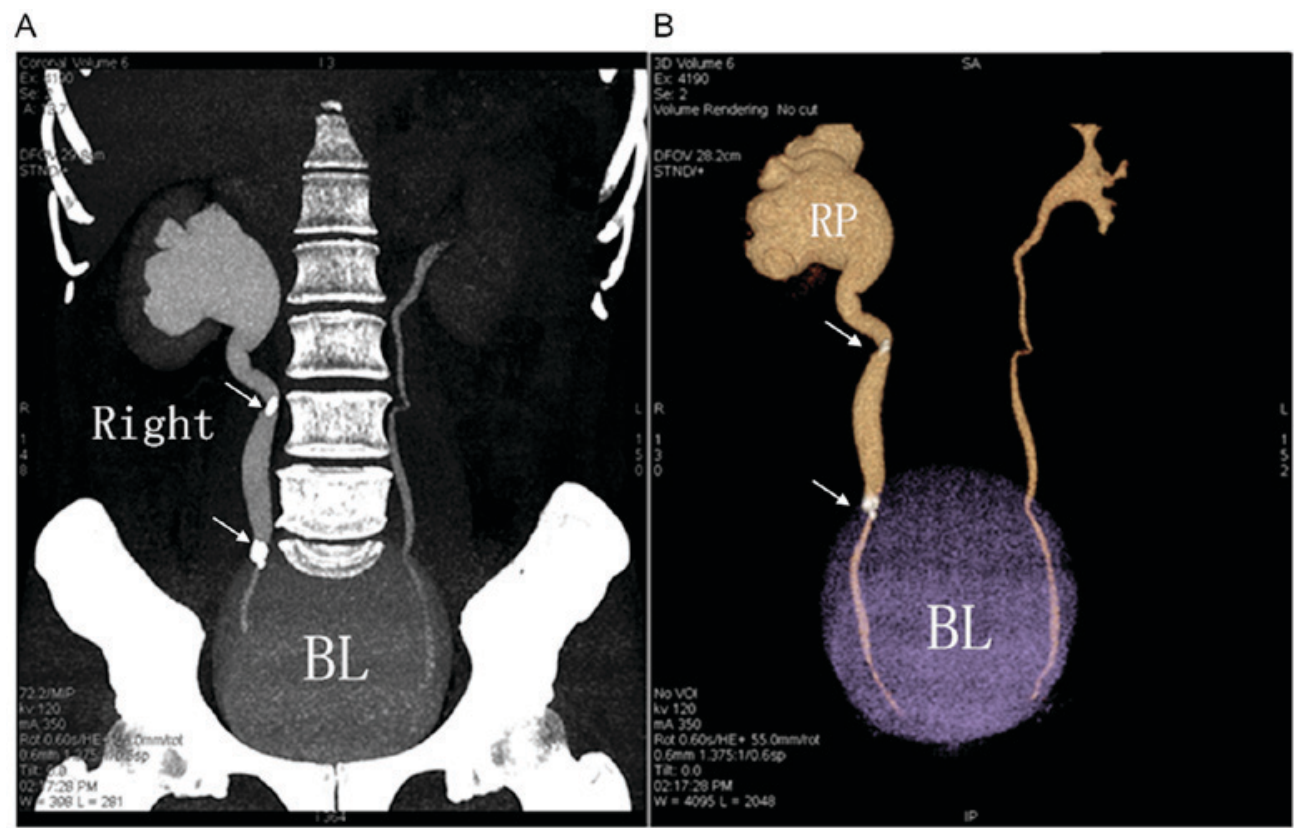

Figure 1. Right side ureteral calculi. Middle and upper ureteral calculi (arrow in A and B) combined with hydronephrosis and hydroureter on the right side visualized by (A) maximum intensity projection and (B) volume rendering. BL, bladder; RP, renal pelvis.

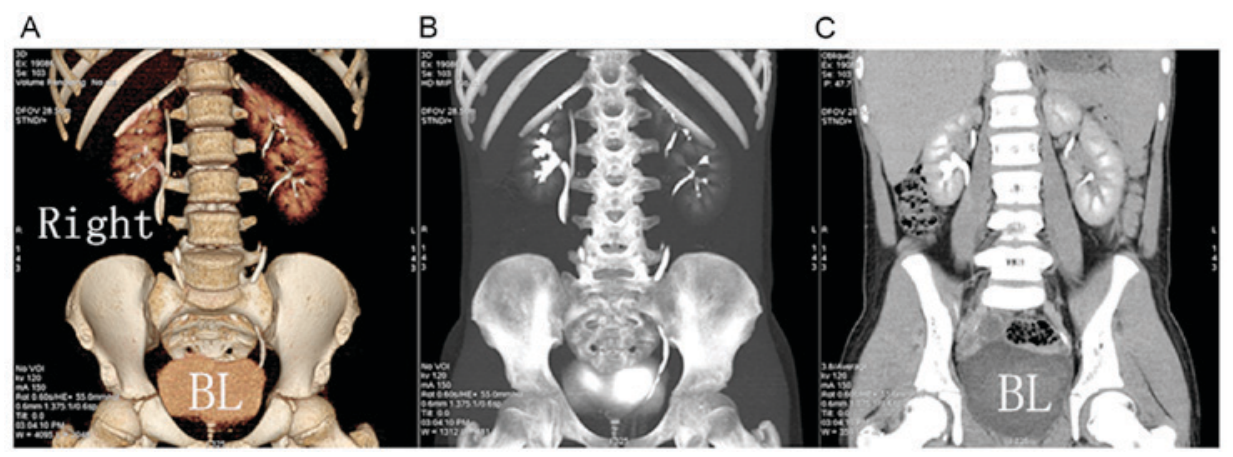

Figure 2. Bilateral double renal pelvis and ureter malformation. Bilateral double renal pelvis and ureter malformation, mild dilation and hydrops of the right pelvis and ureter, visualized by (A) volume rendering, (B) maximum intensity projection and (C) multiplanar reformations. BL, bladder.

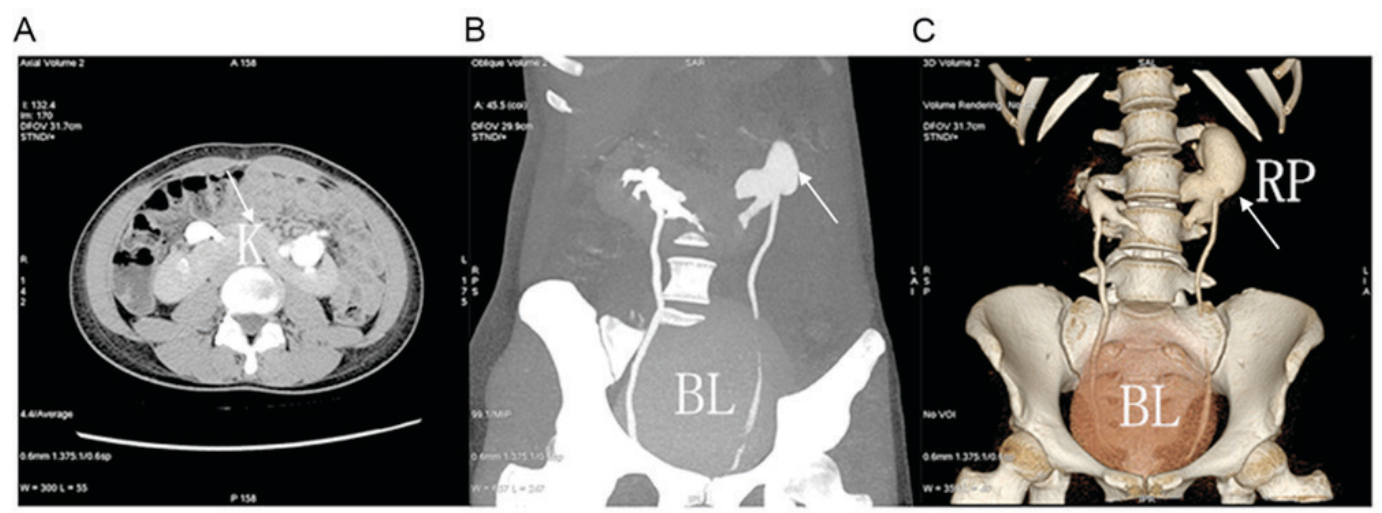

Figure 3. Horseshoe kidney malformation. Adhesion of lower poles of the kidneys (arrow in A), obvious dilation and hydrops of the left kidney (arrows in B and $\mathrm{C}$ ), as visualized by $\mathrm{BL}$, bladder; $\mathrm{U}$, ureter; $\mathrm{K}$, kidney; RP, renal pelvis.

are widely available (10). However, because of the inherent physical properties of these two techniques, their detection rate of urinary tract obstruction remains low (10). Certain researchers advocate the use of abdominal compression during
CT scanning of the urinary tract $(11,12)$. However, abdominal compression is unfavorable for maintaining the integrity of the urinary system (12), particularly in patients with urinary tract obstruction, where the retention of the contrast agent in 


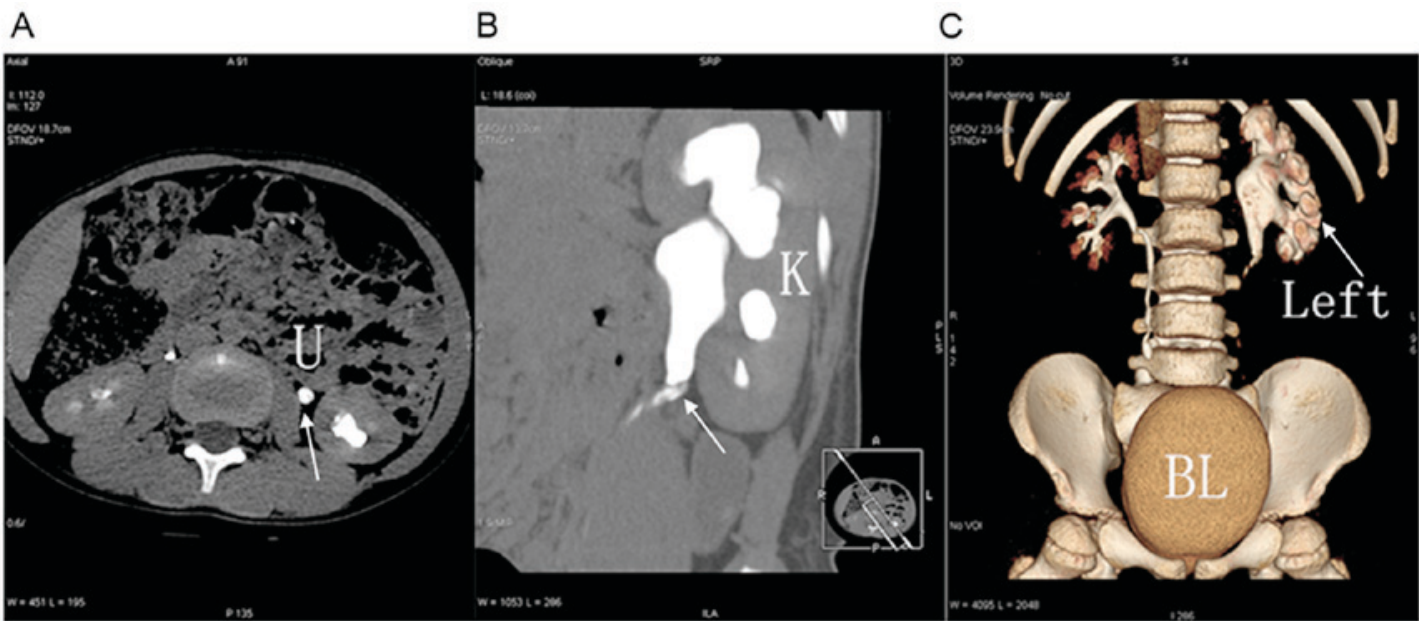

Figure 4. Ureteral valve diseasewas indicated in the left renal pelvis and the ureteropelvic junction (arrow in A and B), and obvious dilation, hydrops and obstruction of the left kidney (arrow in C), visualized by (A) multiplanar reformations, (B) maximum intensity projection and (C) volume rendering. BL, bladder; U, ureter; K, kidney.

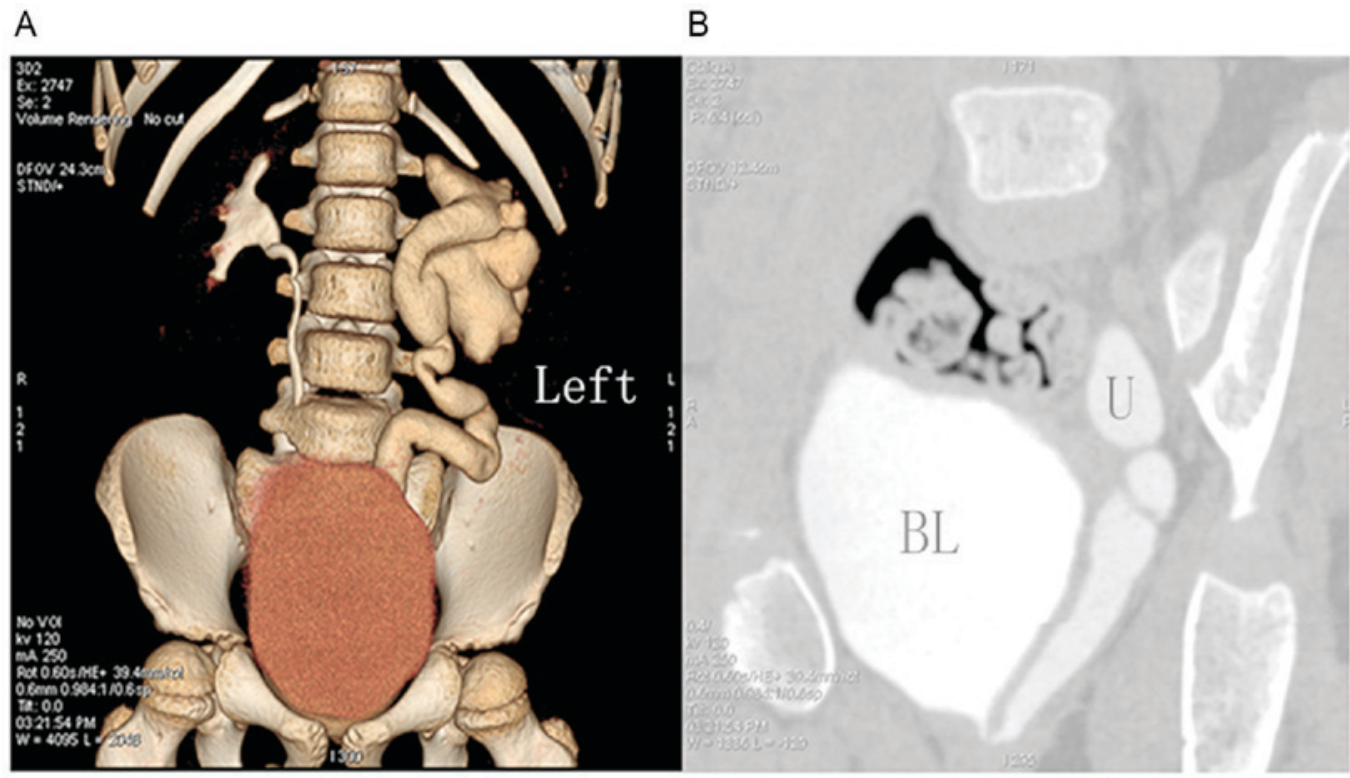

Figure 5. Left side congenital megaureter. (A) volume rendering and (B) multiplanar reformations indicating tortuosity and dilation of the full length of the right ureter and ureteral stricture. BL, bladder; $\mathrm{U}$, ureter.

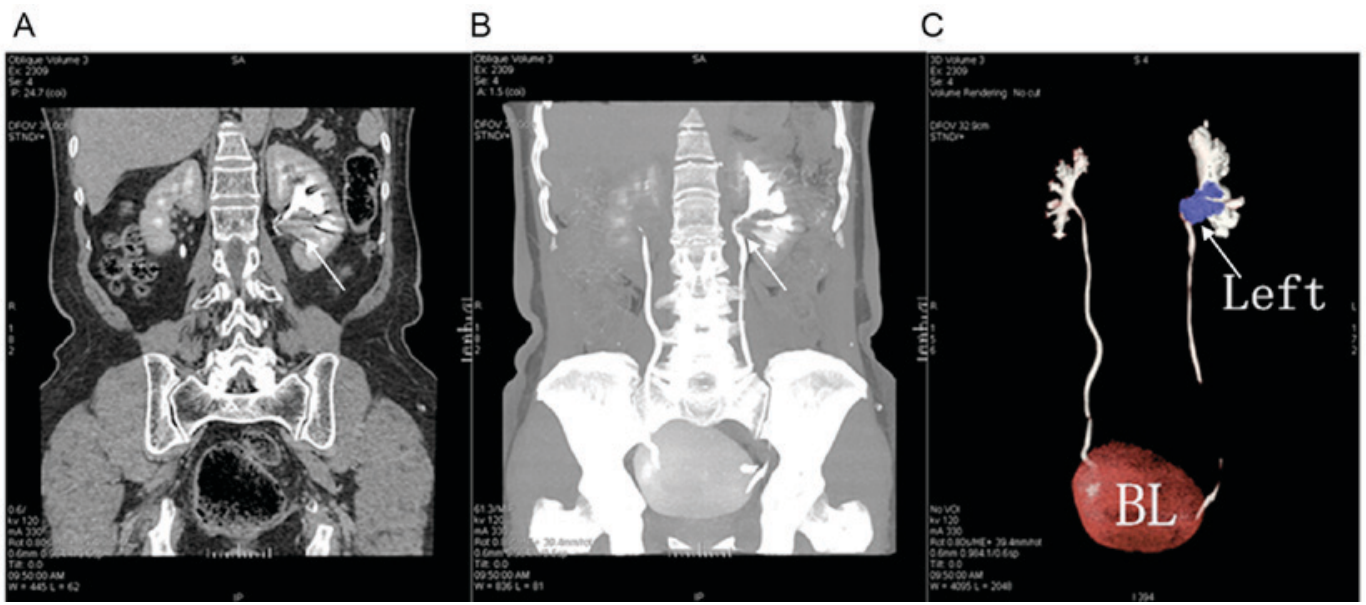

Figure 6. Left pelvis tumor combined with left hydronephrosis. Left pelvis tumor (arrow in B and C) and left hydronephrosis, as visualized by (A) multiplanar reformations, (B) maximum intensity projection and (C) volume rendering. BL, bladder. 
B

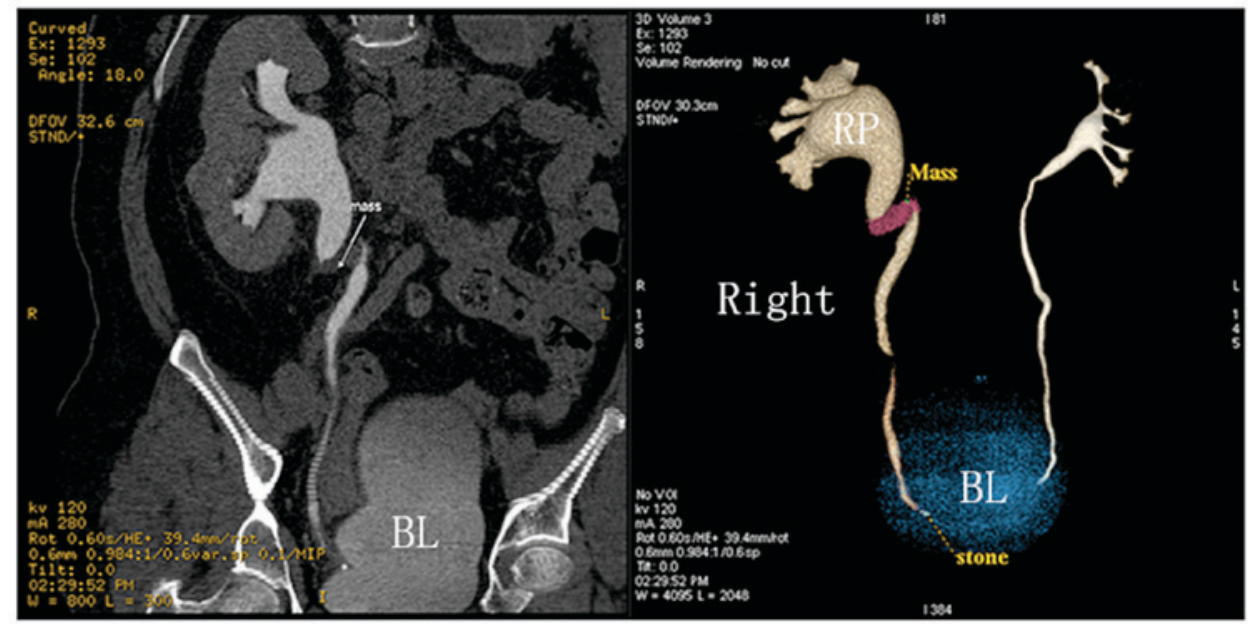

Figure 7. Right upper ureteral tumor. (A) Right upper ureteral tumor visualized by MRP. (B) VR of the right upper ureteral tumor, and hydrops of the upper ureter and kidney. RP, renal pelvis; BL, bladder.

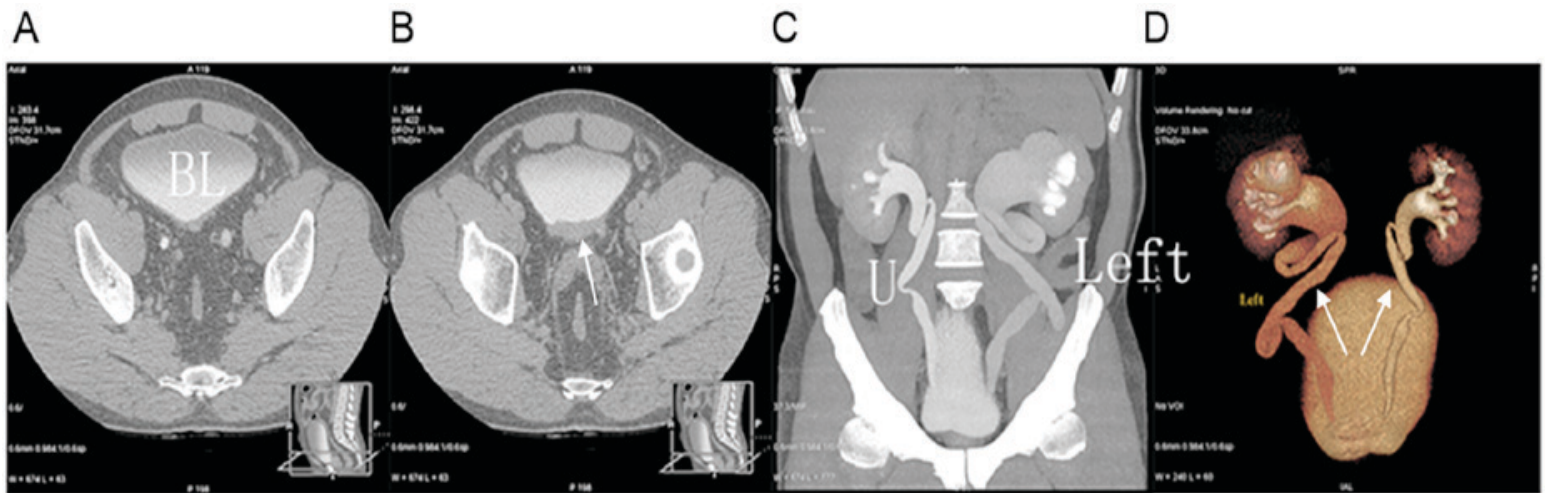

Figure 8. Bladder tumor. (A and B) Different scanning slices of multiplanar reformations indicating a tumor at the trigone of the urinary bladder (arrow in B). (C) Maximum intensity projection and (D) volume rendering indicating tortuosity and elongation of the bilateral ureters, and bilateral hydronephrosis (arrow in D). BL, bladder; U, ureter.

the ureter requires a lack of compression. Of the 46 patients with urinary tract obstruction included in the present study, the obstructed ureter was fully displayed in the first CT scan during excretory phase with good continuity in $96 \%$ of cases. In the remaining $4 \%$ patients with ureter obstruction, obstruction was successfully indicated after the second excretory phase CT scan.

The present study was limited by a small sample size and the absence of an objective estimate of the scan start time for excretory phase. For 2 patients with severe hydronephrosis, the scan of the excretory phase started too early and satisfactory excretory phase images were only obtained at the correct time during the second scan. Furthermore, tumors were scanned during the excretory phase without staging or blood supply assessment. For 1 patient with a pelvis tumor leading to hydronephrosis, scanning during the excretory phase failed to detect the presence of tumor thrombi in the renal vein. The tumor staging was performed through multi-phase scanning.

In conclusion, the results of the present study indicate that CT scanning of the urinary tract during the excretory phase is easy to implement, Single scan radiation dose is small and imaging time is short. The excretory phase CT scanning can assess the obstruction site and nature of obstruction, and has diagnostic value for urinary tract obstruction.

\section{Acknowledgements}

The present study was supported by the Young and Middle-aged Scientific Research Innovation Fund of the Second Affiliated Hospital of Harbin Medical University (grant no. CX2016-01).

\section{References}

1. Stacul F, Rossi A and Cova MA: CT urography: The end of IVU? Radiol Med 113: 658-669, 2008.

2. Van Der Molen AJ, Cowan NC, Mueller-Lisse UG, Nolte-Ernsting CC, Takahashi S and Cohan RH: CT urography: Definition, indications and techniques. A guideline for clinical practice. Eur Radiol 18: 4-17, 2008.

3. Kemper J, Regier M, Stork A, Adam G and Nolte-Ernsting C: Improved visualization of the urinary tract in multi detector CT urography (MDCTU): Analysis of individual acquisition delay and opacification using furosemide and low-dose test images. J Comput Assist Tomogr 30: 751-757, 2006.

4. Dalla Palma L, Morra A and Grotto M: CT-urography. Radiol Med 110: 170-178, 2005 (In Italian). 
5. Yang Q, Zhang ZR, Liu BL, Wang SH and Sui BB: Clinical evaluation of MSCTU in the diagnosis of upper urinary tract obstruction. J Clin Radiol 25: 256-259, 2006.

6. Nawfel RD, Judy PF, Schleipman AR and Silverman SG: Patient radiation dose at $\mathrm{CT}$ urography and conventional urography. Radiology 232: 126-132, 2004.

7. He YQ, Tang BH, Li LC, Wu RG, Huang DC, Liang JX and Dong CL: Multi-slice spiral CT urography in the diagnosis of urinary congenital abnormities. Chin J Radiol 40: 853-856, 2006.

8. Pan WL and Wang XT: Clinical evaluation of Multi-slice spiral CT urography in the diagnosis of Pyelic and Uretal disease. J Pract Radiol 12: 1655-1658, 2007.

9. Ma ZL, He LY, Yan YC, Lv XH, Liu SY and Cao BX: Application of low-dose excretory CT urography. J China Clin Med 6: 484-496, 2009
10. EI-Ghar ME, Shokeir AA, EI-Diasty TA, Refaie HF, Gad HM and EI-Dein AB: Contrast enhanced spiral computerized tomography in patients with chronic obstructive uropathy and normal serum creatinine: A single session for anatomical and functional assessment. J Urol 172: 985-988, 2004.

11. Kong WF, Liu RB, Xing Y, Wang N and Shang L: Optimization of 64-detecter CT urography of normal upper urinary tract: Effect of compression, prolongation of acquisition delay, and oral water on images. J Med Imaging 4: 443-446, 2009.

12. Chow LC and Sommer FG: Multi detector CT urography with abdominal compression and three-dimensional reconstruction. AJR Am J Roentgenol 177: 849-855, 2001. 